3. Porter RS, Zhao N. Patterns of injury in belted and unbelted individuals presenting to a trauma centre after motor vehicle crash: seat belt syndrome revisited. Ann Emerg Med. 1998;32:418-24.

A. Ambrós Checa, S. García-Velasco Sánchez-Morago* y H. Abdel-Hadi Álvarez

Servicio de Medicina Intensiva, Hospital General de Ciudad Real, Ciudad Real, España
* Autor para correspondencia. Correo electrónico: santgarc@gmail.com (S. García-Velasco Sánchez-Morago).

doi:10.1016/j.medin.2010.09.005

\section{Experiencia con tigeciclina en el tratamiento de gérmenes multirresistentes en $\mathrm{UCl}$}

\section{Experience with tigecycline in the treatment of multiresistant germs in the ICU}

\section{Sr. Director:}

La tigeciclina es un antibiótico perteneciente al grupo de las glicilglicinas, con potente actividad frente a una amplia variedad de microorganismos, incluidos los multirresistentes ${ }^{1}$. Actúa bloqueando la síntesis proteica mediante la fijación del complejo aminoácido-ARN al ribosoma.

Aunque las indicaciones aprobadas por la Food and Drug Administration (FDA) no son idénticas a las de la European Medicines Agencie (EMEA) ${ }^{1}$, su uso en nuestro país está limitado a las indicaciones avaladas por los estudios clínicos realizados, que consisten básicamente en infecciones intraabdominales e infecciones complicadas de piel y tejidos blandos. Sin embargo en la práctica clínica, su utilización se amplía para el tratamiento de infecciones graves por gérmenes multirresistentes ${ }^{2,3}$.

Hemos analizado durante el pasado año 2009 las indicaciones y resultados del tratamiento con tigeciclina en la $\mathrm{UCl}$ de un hospital de tercer nivel. Para ello, se definió la respuesta clínica como positiva cuando hubo mejoría parcial o total de signos y síntomas de infección, negativa cuando no hubo mejoría o hubo deterioro clínico, e incierta cuando no hubo datos que nos permitieron conocerla.

La respuesta microbiológica se consideró positiva cuando se negativizaron los cultivos de control a partir del $5^{\circ}$ día de tratamiento, negativa cuando persistía el microorganismo a pesar de tratamiento correcto, y no documentada cuando no se realizaron cultivos de control.

Se realizaron 44 tratamientos con tigeciclina durante al menos 1 semana, en pacientes de 48,1 $\pm 15,5$ años, siendo el $81,8 \%$ hombres. La gravedad de los pacientes al ingreso, medida como mediana (y rango intercuartílico) de APACHE fue 22 (16-28). El motivo de ingreso fue por patología médica en $43,2 \%$, trauma en $29,5 \%$ o quirúrgica en $27,3 \%$. Hasta un $82 \%$ no tuvieron comorbilidades importantes.

La localización más frecuente de la infección fue del tracto respiratorio $(51,2 \%)$ siendo el $27,3 \%$ neumonía asociada a ventilación mecánica, traqueobronquitis $20,4 \%$ y neumonía de la comunidad en $2,3 \%$. Otras localizaciones menos frecuentes fueron piel y herida quirúrgica en $13,6 \%$, intraabdominal en $13,6 \%$, bacteriemia primaria en $9,1 \%$ e infección por catéter en 4,5\%. Las indicaciones fueron según ficha técnica únicamente en un $27,9 \%$ y en un $9,3 \%$ se usó de forma empírica.

El germen para el que se utilizó fue Acinetobacter baumanii $(81,4 \%)$, Klebsiella pneumoniae $(4,7 \%)$ y Enterococcus faecalis $(4,7 \%)$.

El perfil de resistencias del acinetobacter en nuestra unidad se ha ido modificando durante los últimos años, pero globalmente podemos decir que tenemos un $83,8 \%$ de resistencias a carbapenemes y un 74,4\% a ampicilina-sulbactam. Los fármacos testados con menos resistencias, son la amikacina $(9,3 \%)$, colistina $(7,6 \%)$, la y la tigeciclina $(4,2 \%)$.

En al menos el $74,4 \%$ se utilizó en combinación con otro antibiótico: colistina intravenosa en $25,6 \%$, colistina aerosolizada en $32,6 \%$ o amikacina en el $14 \%$, siendo datos muy parecidos a los publicados por Alvarez Lerma et al recientemente 4

Se observó una respuesta clínica positiva en el 67,4\% y negativa en el $30,2 \%$, aunque la respuesta microbiológica fue peor (37,2\% negativizó cultivos, 34,9\% persistieron cultivos positivos y carecemos de datos en $27,9 \%$ ). En ningún caso de persistencia microbiológica se objetivó la aparición de resistencia a tigeciclina.

Como conclusión podemos extraer que la tasa de uso compasivo de tigeciclina en nuestro centro (fuera de las indicaciones aprobadas) ha sido muy elevada, y que ha supuesto una nueva arma de gran ayuda contra gérmenes multirresistentes en entornos de cuidados intensivos, generalmente en asociacion con colistina, como tratamiento de rescate o como uso compasivo, mostrando una buena respuesta clínica y en algunos trabajos, un descenso de la mortalidad $^{5}$.

\section{Bibliografía}

1. Reinert RR, Low DE, Rossi F, Zhang X, Wattal C, Dowzicky MJ. Antimicrobial susceptibility among organisms from the Asia/Pacific Rim. Europe and Latin and North America collected as part of TEST and the in vitro activity of tigecycline. J Antimicrob Chemother. 2007;60:1018-29.

2. Betriu C, Rodriguez-Avial I, Lopez F, Picazo JJ. Comparative study of the in vitro activity of tigecycline against multiresistant Enterococcus faecium isolates. Enferm Infecc Microbiol Clin. 2009;27:302-3. 
3. Sorlozano A, Gutierrez J, Roman E, de Dios LJ, Roman J, Liebana $\mathrm{J}$, et al. A comparison of the activity of tigecycline against multiresistant clinical isolates of Staphylococcus aureus and Streptococcus agalactiae. Diagn Microbiol Infect Dis. 2007;58:487-9.

4. Alvarez Lerma F, Blanco L, Rodriguez JA, Grau S, Conde Estevez $D$, Luque $S$. Diferencias en el uso de tigeciclina entre pacientes críticos y no críticos. Rev Esp Quimioter. 2010;23:63-71.

5. Swoboda S, Ober M, Hainer C, Lichtenstern C, Seiler C, Wendt $\mathrm{C}$, et al. Tigecycline for the treatment of patients with severe sepsis or septic shock: a drug use evaluation in a surgical intensive care unit. J Antimicrob Chemother. 2008;61:729-33.
E. Curiel Balsera*, A.M. Poullet Brea y

M.Á. Prieto Palomino

Unidad de Cuidados Intensivos, Hospital Regional

Universitario Carlos Haya, Málaga, España

* Autor para correspondencia.

Correo electrónico: emiliouci@telefonica.net

(E. Curiel Balsera).

doi:10.1016/j.medin.2010.09.003

\section{Hemorragia cerebelosa secundaria a fuga de líquido cefalorraquídeo tras cirugía del canal lumbar}

\section{Cerebellar hemorrhage secondary to cerebros- pinal fluid leak after lumbar canal surgery}

\section{Sr. Director:}

La hemorragia cerebelosa a distancia (HCD) se considera una complicación rara de la cirugía intracraneal y excepcional tras la intervención espinal ${ }^{1}$. Presentamos el caso de una mujer que desarrolla una HCD en el postoperatorio de estenosis de canal lumbar.

Se trata de una mujer de 58 años de edad, con antecedentes de síndrome depresivo, que es intervenida de estenosis de canal lumbar, realizándosele una laminectomía L5-S1 que se complica con un desgarro de la duramadre, con salida de líquido cefalorraquídeo (LCR), que obliga a su sutura. A las 36 horas de la cirugía, y estando en tratamiento con analgésicos mórficos y sedantes, presenta depresión del nivel de conciencia que revierte completamente con naloxona y flumazenil intravenosos. A las 12 horas de este episodio, y ya sin los fármacos previamente descritos, vuelve a presentar fluctuaciones de la conciencia y cefalea, por lo que se realiza un tomografía computarizada cerebral (TC) que muestra una pequeña hemorragia lineal y otra mayor $(1,4$ $x 0,9 \mathrm{~cm}$ ) en el hemisferio cerebeloso derecho e izquierdo respectivamente. Hemodinámicamente se mantiene estable y la bioquímica, hemograma y coagulación muestran valores normales. La paciente se traslada a un centro hospitalario con servicio de neurocirugía, en donde evoluciona satisfactoriamente con tratamiento conservador.

La HCD se ha descrito tras cirugía supratentorial, presentando una incidencia que varia desde el $0,2 \%$ hasta alcanzar el 3,5-4,9\%, en el caso de intervenciones de aneurismas y después de lobectomías temporales, siendo incluso menor y observándose ocasionalmente después de cirugía raquídea, aunque la tasa real probablemente sea superior al infradiagnosticarse los casos asintomáticos ${ }^{1-4}$. Aunque la etiopatogenia está sujeta a discusión, pudiendo estar involucradas alteraciones de la coagulación, hipertensión arterial y rotación de la cabeza dificultando el drenaje venoso cerebral, la mayoría de autores lo atribuyen a un síndrome de hipotensión de LCR, secundario a pérdida del mismo tras la lesión dural, que secundariamente produciría un descenso del cerebelo con tracción y obstrucción sobre las venas del puente y finalmente un infarto venoso hemorrágico ${ }^{1-4}$. Característicamente las hemorragias son bilaterales y se localizan preferentemente en el vermis y surcos cerebelosos, como en nuestro caso, revelando a veces la TC cerebral una imagen curvilínea hemorrágica en dichos surcos, conocido como el signo de la cebra ${ }^{4,5}$. Clínicamente cursan con cefalea, que no mejora claramente con el decúbito, alteraciones de la conciencia y a veces se acompañan de signos neurológicos focales. El pronóstico depende fundamentalmente de la edad y de la gravedad de la hemorragia, quedando sin o con discretas secuelas neurológicas más del $50 \%$ de casos, llegando a alcanzar una mortalidad del 10 $15 \%{ }^{1}$. El tratamiento varía desde una actitud conservadora hasta cirugía intracraneal urgente, debiendo comprobarse sin demora si existe fuga de LCR y en su caso proceder a reparar urgentemente la fuga dural.

Concluimos haciendo hincapié en que la presentación de cefalea acompañada a veces de fluctuaciones del nivel de conciencia, con o sin disfunción cerebelosa o de tronco, en el postoperatorio de cirugía raquídea, obliga a realizar urgentemente una TC craneal para descartar una HCD.

\section{Bibliografía}

1. Brockmann MA, Groden C. Remote cerebellar hemorrhage: a review. Cerebellum. 2006;5:64-8.

2. Riera Sagrera M, Bergadá García J. Hemorragia cerebelosa tras cirugía espinal. Med Intensiva. 2008;32:54-5.

3. Karaeminogullari O, Atalay B, Sahin O, Ozalay M, Demirors H, Tuncay $C$. Remote cerebellar hemorrhage after a spinal surgery complicated by dural tear: Report and literatura review. Neurosurgery. 2005;57(Supl 1):E215.

4. Nam TK, Park SW, Min BK, Hwang SN. Remote cerebellar hemorrhage after lumbar spinal surgery. J Korean Neurosurg. 2009;46:501-4.

5. Brockmann MA, Nowak G, Reusche E, Russlies M, Petersen D. Zebra sign: cerebellar bleeding pattern characteristic of cerebrospinal fluid loss. J Neurosurg. 2005;102:1159-62.

M.A. Blasco Navalpotro*, F. Del Nogal Sáez y R. Díaz Abad

Servicio de Medicina Intensiva, Hospital Universitario Severo Ochoa. Leganés, Madrid, España

* Autor para correspondencia. Correo electrónico: mblasco.hsvo@salud.madrid.org (M.A. Blasco Navalpotro).

doi:10.1016/j.medin.2010.09.008 